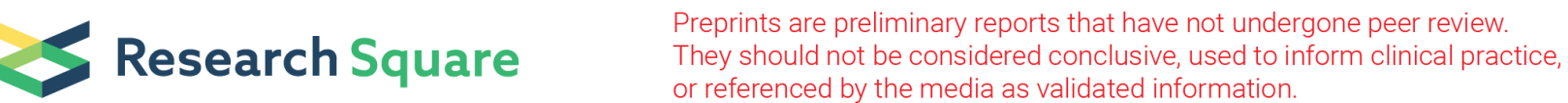

\section{Correlation of sTILs Infiltration and UBR5 Expression on the Efficacy of Neoadjuvant Chemotherapy Efficacy and Prognosis in Early Triple Negative Breast Cancer}

\author{
Jian Pang \\ Xiangya Hospital Central South University \\ Li Zhou \\ Xiangya Hospital Central South University \\ Liqiu Liao \\ Xiangya Hospital Central South University \\ Shouman Wang ( $\sim$ pn201711@163.com) \\ Central South University
}

\section{Research article}

Keywords: Triple negative breast cancer, UBR5 gene, Tumor infiltrating lymphocytes, Neoadjuvant chemotherapy, Bioinformatics analysis

Posted Date: January 13th, 2021

DOI: https://doi.org/10.21203/rs.3.rs-142859/v1

License: (c) (1) This work is licensed under a Creative Commons Attribution 4.0 International License.

Read Full License 


\section{Abstract}

Neoadjuvant chemotherapy (NAC) is widely used in patients with TNBC, but there are significant differences in the efficacy of chemotherapy in different patients. Stromal tumor infiltrating lymphocytes (sTILs) have been widely studied as biomarkers reflecting the therapeutic effect of NAC. As a tumorassociated gene, UBR5 has been found to be associated with chemotherapy sensitivity and prognosis of many cancers. This is the first time that we have combined UBR5 and STILs to evaluate the chemotherapy efficacy of patients with TNBC treated with NAC and to study the correlation between sTILs and UBR5.

Methods

SPSS was used to analyze the correlation between UBR5 and STILs and clinicopathological data. Then doxorubicin and mitoxantrone treated mouse breast cancer cells to explore the relationship between UBR5 expression and chemotherapeutic drug sensitivity. Finally, $\mathrm{R}$ language analyzes the relationship between UBR5 and immune-related genes and immune score.

Results

In patients with TNBC treated with NAC, the expression level of UBR5 and the degree of infiltration of sTILs are independent factors that affect whether the patient can achieve PCR and the expression between the two is negatively correlated. UBR5 expression can cause mouse breast cancer cells to develop resistance to doxorubicin and mitoxantrone. At the same time, UBR5 is also highly correlated with a variety of immune-related genes and immune scores.

\section{Conclusion}

UBR5 and sTILs can jointly predict the efficacy of NAC in patients with TNBC. At the same time, the expression of UBR5 is also an important reason for chemotherapy resistance in patients with TNBC.

\section{Background}

Triple negative breast cancer (TNBC) is a highly metastatic and invasive subtype of breast cancer with a poor overall prognosis ${ }^{[1]}$. Because TNBC does not express human epidermal growth factor receptor 2 , estrogen receptor and progesterone receptor (HER2, ER and PR, respectively) ${ }^{[2]}$, chemotherapy is still the mainstay treatment method for most patients with TNBC ${ }^{[1]}$. In addition, neoadjuvant chemotherapy (NAC) is widely used to treat these patients, one-third of which can be treated with standard NAC to achieve pathological complete remission ${ }^{[3]}$ (PCR). However, due to the high degree of malignancy of TNBC, many patients who have undergone standard NAC treatment still have tumor progression during treatment, and even lose the opportunity for surgery. Therefore, it is particularly important to be able to evaluate the efficacy of NAC early and to screen out patients with poor chemotherapy sensitivity for intensive treatment. 
Due to the genomic instability of TNBC and high tumor mutation burden, the degree of infiltration of stromal tumor infiltrating lymphocytes (sTILs) in TNBC is higher than that of other breast cancer subtypes ${ }^{[4,5]}$. sTILs play an important role in the anti-tumor immune response and have been widely studied as biomarkers for predicting the prognosis of patients with TNBC and the efficacy of chemotherapy in patients receiving NAC ${ }^{[6]}$.

The UBR5 gene is a member of the E3 ubiquitin ligase family and is also a tumor-related gene, which has received extensive attention in recent years ${ }^{[7]}$. Data from the TCGA database shows that UBR5 is abnormally expressed in many types of cancers and is closely involved in various cellular functions, such as DNA damage repair, immune response, transcription regulation, and the cell cycle ${ }^{[8-11]}$. UBR5 is also related to chemotherapy sensitivity and the prognosis of various cancers, such as prostate cancer and ovarian cancer ${ }^{[12-14]}$. However, little is known about the expression levels of UBR5 in patients with TNBC. Additionally, whether UBR5 expression in cancer tissues of patients with TNBC is associated with the chemotherapy sensitivity and prognosis remains unclear. It is also unclear whether UBR5 expression and the degree of infiltration of sTILs in puncture specimens can be used to evaluate the efficacy of NAC and prognosis. To address this gap, we explored the correlation between the expression level of UBR5 and the degree of sTILs infiltration in clinical patients with TNBC to evaluate the efficacy of NAC treatment and the prognosis of patients. At the same time, in cell experiments, we also conducted experiments on the influence of UBR5 expression level on the sensitivity of chemotherapy drugs. Finally, we used bioinformatics analysis to find the genes related to UBR5 expression and immune cell infiltration and the relationship between UBR5 expression and immune score.

\section{Method}

\section{Patient Background}

Our study included 185 pathologically confirmed patients with TNBC who received systematic treatment in Xiangya Hospital of Central South University from April 2015 to April 2019. Exclusion criteria included a past history of breast cancer, breast cancer during pregnancy, and breast cancer patients with distant metastases at the first diagnosis. Patients with acute or chronic injuries (inflammation), blood diseases, and metabolic diseases were also excluded. We collected patient clinical pathological data, including tumor size, lymph node staging, histological grade, chemotherapy regimen, and proliferation index (KI$67 \%$ ). Among these patients, 81 patients with TNBC received 4 cycles of NAC therapy, mainly based on anthracyclines. PCR was defined as patients with no invasive tumor or only residual ductal carcinoma in situ (DCIS) of the breast. All patients received surgical treatment (modified radical mastectomy or radical surgical resection of breast cancer plus sentinel lymph node biopsy). Disease free survival (DFS) is the time between the disease progression or the last follow-up time and the day of surgery. The last follow-up time was August 2020. Patient follow-up lasted for 3 to 64 months (median 33 months), wherein 46 patients experiences recurrence or distant metastasis. The average time for recurrence/metastasis was 11.9 months. The study was approved by the ethics committee of Xiangya Hospital. 


\section{sTILs Score}

We selected the puncture specimen of the patient's first visit to determine the degree of sTILs infiltration. sTILs are scored based on the percentage of lymphocytes in the stromal area, and the score does not consider the area occupied by cancer cells. STILs also do not include tumor infiltration, DCIS, and lymphocytes at the border of normal lobules ${ }^{[5]}$. The scoring criteria for STILs was as follows: (a) Low level of sTILs infiltration (0-10\% sTILs); (B) High level of sTILs infiltration (> 10\% sTILs) (Figure 2).

\section{Immunohistochemistry}

Postoperative specimens were collected for the detection of UBR5 protein in cancer cells and CD8 protein in T cells. Paraffin-embedded specimens from patients with TNBC were obtained from the Department of Pathology, Xiangya Hospital, Central South University. Monoclonal antibodies against UBR5 (Abcam, ab70311) and CD8 (Boster, BM4379) were used for immunohistochemical analysis. The tissue section (5 $\mathrm{mm}$ ) was deparaffinized in xylene and rehydrated with distilled water. EDTA (ZSGB-BIO, pH 9.0) was used for antigen retrieval, and $3 \% \mathrm{H} 2 \mathrm{O} 2$ was used to remove endogenous peroxidase. After incubating at $4{ }^{\circ} \mathrm{C}$ overnight, the antigen-antibody reaction was detected with a secondary antibody kit (ZSGB-BIO, PV9001), and finally the tissue slide was counterstained with hematoxylin, dehydrated, and fixed. The section without primary antibody was used as a negative control for staining. Two pathologists independently performed immunohistochemical scoring. At 400X magnification, five areas of each slice were randomly selected. One of the criteria for the positive expression of UBR5 is the appearance of transparent brown particles in the cytoplasm or nucleus of cancer cells. One of the criteria for positive CD8 expression is that transparent yellow particles can be seen on the mesenchymal T cell membrane. In order to semiquantitatively analyze the immunostaining results of UBR5 and CD8, the product of the staining intensity score and the percentage of positive cells was selected as the final result. The staining intensity was scored from 0 to 3 ( 0 , none; 1 , weak; 2 , moderate; 3 , strong), and the percentage of positive cells was scored from 0 to 4 ( 0 , none; $1,<10 \%$; 2 . From $10 \%$ to $50 \%$; 3 . From $51 \%$ to $80 \% ; 4$.> $80 \%$ ). A final score of $0-1$ is considered low expression; a score greater than 1 is considered high expression.

\section{Drug Sensitivity Test}

The cell lines used in this study were obtained from the ATCC cell bank. Drug sensitivity experiments were performed with the 4T1/GFP mouse breast cancer cell line and the existing 4T1/Ubr5 $5^{-/-}$cell line with stable UBR5 knockout. Briefly, $5 \times 10^{3}$ cells per well were seeded into $96-$ well tissue culture plates. After overnight culture, different concentrations of the chemotherapeutic drugs, Doxorubicin or Mitoxantrone, were added to the medium. Following incubation for 72 hours, the cell viability was measured by the sulforhodamine B (SRB) assay as follows: the cells were fixed with $10 \%$ trichloroacetic acid at $4^{\circ} \mathrm{C}$ for 30 minutes, and then stained with $0.4 \%(\mathrm{w} / \mathrm{v})$ SRB in $1 \%$ acetic acid solution for 30 minutes. Then, the SRB was removed, and the tissue culture plate was washed with $1 \%$ acetic acid for 5 minutes. Finally, $10 \mathrm{mM}$ Tris buffer was used to dissolve the bound SRB, and the absorbance (OD) was measured at $510 \mathrm{~nm}$ on a 
microplate reader. The half of the maximum inhibitory concentration (IC50) was deteremined based on the growth inhibition curve.

\section{Bioinformatics Analysis}

The original mRNA expression data of breast cancer was downloaded from the TCGA database (https://portal.gdc.cancer.gov/). A total of 1222 specimens were analyzed, including 113 normal specimens and 1109 cancer specimens. Using sSGSEA, the expression levels of all genes in the selected TNBC samples were first sorted to obtain their rank among all genes. Then, we find and count the genes present in the expression data of immune cell infiltration and immunoregulatory pathways from all genes and sum the expression levels of these genes. Then, based on the above evaluation, the enrichment score of each gene in the pathway was calculated. The gene order was disrupted in order to recalculate the enrichment score and repeated a thousand times, ultimately yielding a $p$ value according to the distribution of the gene enrichment score and integrate the final enrichment score of the relevant gene set. For the candidate genes that affect immune infiltration in the sample, we used the $\mathrm{R}$ language to screen candidate genes related to UBR5. The condition of the correlation threshold filter is that the absolute value of the correlation coefficient is greater than 0.3 and $p<0.001$. Finally, the immune score and stromal score are calculated based on the ESTIMATE algorithm, and the correlation coefficient between UBR5 expression and immune score is calculated and a scatter diagram is drawn.

\section{Statistical Analysis}

Pearson's chi-squared test or Fisher's exact test were used to determine the relationship between UBR5 expression, STILs, and other indicators and clinicopathological parameters of patients with TNBC. The Kaplan-Meier curve was used to compare survival results, and the Cox regression model was used to compare prognostic factors related to DFS. The correlation between the two variables was evaluated by the Spearman rank correlation coefficient test. A $p$ value $<0.05$ or $<0.001$ was considered statistically significant. Statistical analysis was performed using SPSS version 23.0.

\section{Results}

\section{Inclusion of Patient Information}

We initially enrolled 199 patients with TNBC in our study, but excluded 3 patients with breast cancer during pregnancy, 6 patients with distant metastasis at first diagnosis, and 5 patients with acute and chronic inflammation. Finally, 185 patients with TNBC were included in our study. Among them, 81 patients received 4 cycles of NAC treatment, and 13 patients achieved PCR (Figure 1).

\section{The relationship between UBR5 expression, sTILs and clinicopathological factors}

From the 185 cases of TNBC, including 44 cases of low UBR5 expression and 141 cases of high UBR5 expression. The infiltration level of CD8+ T cells is an influencing factor affecting the expression of UBR5 in patients $(p=0.009<0.05$; Table 1$)$. A total of 81 patients with TNBC were treated with NAC, the level of 
CD8+ T cells and sTILs infiltration are factors that affect whether the patients can achieve PCR $(p=0.016$; $\mathrm{p}=0.010$, respectively; Table 1 ).

\section{UBR5 and sTILs are independent factors affecting PCR in patients with TNBC treated with NAC}

In our study, 81 patients with TNBC received NAC treatment. There were 25 patients in the low UBR5 expression group with a PCR rate of $33.3 \%$. There were 56 patients in the high UBR5 expression group with a PCR rate of $9.8 \%$. Patients with low UBR5 expression tended to have better PCR $(p=0.018$; Table 2). There were 52 patients in the low sTILs infiltration group with a PCR rate of $7.7 \%$. There were 29 patients with high sTILs infiltration with a PCR rate of 31.0\%. Patients with high sTILs infiltration tended to have better PCR ( $p=0.010$; Table 2). Multivariate analysis showed that in patients with TNBC treated with NAC, the expression level of UBR5 and the degree of sTILs infiltration were independent factors affecting PCR ( $p=0.039 ; p=0.037$, respectively; Table 3$)$.

\section{Prognostic analysis of patients with TNBC who received NAC treatment and did not reach PCR}

Survival analysis showed that tumor size and lymph node staging were independent factors that influenced the DFS rate of patients ( $p=0.020 ; p=0.010$, respectively). However, the expression of UBR5 and the degree of sTILs infiltration were not independent factors that affected DFS of patients receiving NAC treatment but not achieving PCR.

\section{Prognostic analysis of patients with TNBC}

Our analysis revealed that the DFS rate of patients with high UBR5 expression was significantly lower than that of patients with low UBR5 expression ( $59.5 \%$ vs. $91.7 \%$ ). Compared to patients with low sTILs infiltration, patients with high sTILs infiltration had a significantly higher DFS rate ( $82.8 \%$ vs. $62.6 \%)$. The DFS rate of patients with different tumor sizes $(P=0.011)$ and the DFS rate of patients with different lymph node stages $(P=0.020)$ were also significantly different. In the Cox analysis, UBR5 expression, degree of sTILs infiltration, tumor sizes, and lymph node stages were independent factors that influenced the DFS rate in patients with TNBC $(P<0.05)$ (Table 5).

\section{Correlation analysis between UBR5 and sTILs}

In 185 patients with TNBC, Spearman correlation analysis showed that the expression of UBR5 was negatively correlated with the degree of infiltration of sTILs. $(R=-0.174, p=0.018<0.05)($ Table 6$)$.

\section{Drug sensitivity test}

We used two chemotherapy agents, doxorubicin and mitoxantrone, to conduct drug sensitivity experiments on breast cancer cells. The $4 \mathrm{~T} 1 / \mathrm{Ubr} 5-/$ - cells were 18 times more sensitive to doxorubicin than the 4T1/GFP cells, with an IC50 of $480.24 \mathrm{nM}$ and $27.34 \mathrm{Nm}$, respectively (Figure 3a). The two groups of cells were treated with also treated with the chemotherapeutic drug, mitoxantrone. The 4T1/Ubr5-/- 
were 19 times more sensitive to mitoxantrone than the 4T1/GFP cells, with an IC50 of 0.001401 uM and $0.027504 u M$, respectively (Figure $3 b$ ).

\section{Bioinformatics Analysis}

Bioinformatics analysis of the TNBC samples revealed enrichment of genes related to a variety of immune cell infiltration and immunoregulatory pathways, such as $T$ cell and $B$ cell infiltration and immune checkpoint regulation (Figure 4a). By analyzing the correlation between UBR5 and immune gene expression in the TNBC samples, we found that UBR5 expression was positively correlated with TMEM184C, SMAD2, TGFBRAP1, BMPR2, NFAT5, REL, and SSH1 gene expression $(\nabla r \Downarrow>0.3, p<0.001)$. The expression of UBR5 was negatively correlated with the expression of the genes TNFSF12 and VAMP5 $(\mathbb{B r} \backslash>0.3 \& p<0.001)$ (Figure 4b). The immune score and matrix score calculated based on the ESTIMATE algorithm can promote the quantification of immune and matrix components in tumors. By analyzing the correlation between UBR5 expression and immune scores, we found that the expression of UBR5 is negatively correlated with the three immune scores, StromalScore, ImmuneScore, and ESTIMATEScore (Figure 4c).

\section{Discussion}

The results of the study showed that in patients with TNBC treated with NAC, the expression level of UBR5 and the degree of infiltration of sTILs are independent factors that affect whether the patient can achieve PCR. Drug sensitivity experiments have found that the expression of UBR5 can cause mouse breast cancer cells to develop resistance to doxorubicin and mitoxantrone. In patients with TNBC who received NAC treatment and did not reach PCR, the expression of UBR5 and the degree of STILs infiltration were not independent factors affecting DFS. In patients with TNBC, the independent factors influencing DFS were UBR5 expression, degree of sTILs infiltration, lymph node stage, and tumor size ( $P$ $<0.05)$. Spearman correlation analysis showed that the expression of UBR5 in patients with TNBC was negatively correlated with the degree of sTILs infiltration. Bioinformatic analysis revealed that UBR5 is highly correlated with a variety of immune-related genes and immune scores. Numerous studies have proven STILs to be reliable, inexpensive, and easily available immune markers for anti-tumor immunity in breast cancer ${ }^{[6]}$. Our study found that STILs are a prognostic indicator in patients with TNBC and an effective predictor of the efficacy of patients with TNBC receiving NAC. This is consistent with the results of many studies. An analysis of 2148 patients with early TNBC confirmed that high sTILs infiltration has

better prognostic value ${ }^{[15]}$, and the results of other studies also support this conclusion ${ }^{[16-19]}$. In patients with TNBC treated with NAC, high sTILs infiltration is highly correlated with better PCR in patients ${ }^{[19-22]}$. There are similar findings in early HER2-positive breast cancer. sTILs can be used as a prognostic biomarker to identify early HER2-positive breast cancer with a low risk of recurrence ${ }^{[23]}$. Baseline sTILs in pre-NAC core needle biopsy specimens were significantly correlated with the PCR and prognosis of HER2positive breast cancer. Infiltration of more than $20 \%$ sTILs is a feasible diagnostic indicator for predicting the PCR and prognosis of patients with HER2-positive breast cancer receiving NAC treatment ${ }^{[24]}$. 
Additionally, it has been reported that trastuzumab treatment is more effective with high sTILs infiltration in patients with metastatic HER2-positive breast cancer ${ }^{[23]}$. However, some studies have shown that the increased sTILs infiltration after NAC is related to circulating tumor cell (CTC), which is found to be an unfavorable prognostic factor ${ }^{[25]}$.

UBR5 is also a nuclear phosphate protein with unclear function. UBR5 amplification and overexpression occur in more than $20 \%$ of breast cancer cases and UBR5 overexpression significantly reduces the survival time of patients with breast cancer ${ }^{[14]}$. Simultaneous targeting of UBR5 and PD-L1 in animal models has shown powerful therapeutic benefits for tumor-bearing hosts ${ }^{[14]}$. In breast cancer, UBR5 is coamplified with MYC, thereby limiting MYC-dependent apoptosis of cancer cells ${ }^{[12]}$. In pancreatic cancer, UBR5 downregulates FBP1 by downregulating the expression of C/EBPa, thereby promoting the growth of cancer cells ${ }^{[13]}$. In gallbladder cancer, UBR5 can promote tumor growth by affecting the PTEN/PI3K/Akt signaling pathway ${ }^{[26]}$. In laryngeal cancer, UBR5 regulates the proliferation and radiosensitivity of cancer cells through the p38/MAPK signaling pathway ${ }^{[27]}$. UBR5 overexpression is also a poor prognostic indicator of gastric cancer, colon cancer, and lung cancer ${ }^{[28-33]}$. Our research results show that UBR5 expression is an independent factor that influencs whether patients with TNBC undergoing NAC treatment achieve PCR, and also influences DFS in patients with TNBC. UBR5 expression plays an important role in the occurrence and development of different cancers, and its regulatory network is complex. In different tumors, the high levels of UBR5 expression is often highly correlated with worse prognosis and chemotherapy effect. Therefore, UBR5 is worthy of further research.

While investigating whether the expression of UBR5 affects the drug sensitivity of TNBC cells, we found that the high UBR5 expression group had significant doxorubicin and mitoxantrone resistance. In breast cancer, overexpression of UBR5 stabilizes $\beta$-catenin signaling, leading to poor prognosis and tamoxifen resistance in ER-positive breast cancer ${ }^{[34]}$. In prostate cancer, UBR5 activates Wnt/ $\beta$-Catenin signal transduction, thereby causing docetaxel resistance ${ }^{[35]}$. In ovarian cancer, UBR5 downregulates the expression of MOAP-1, leading to cisplatin resistance in ovarian cancer ${ }^{[36]}$. Therefore, reducing the level of UBR5 may result in improved chemotherapy efficacy for patients with TNBC.

To our knowledge, this is the first time that UBR5 and sTILs have been reported to be independent factors that affect whether patients with TNBC undergoing NAC treatment can achieve PCR, and that they are negatively correlated. Combining these two indicators provides a more accurate basis to predict the efficacy of NAC in patients with TNBC. This has important clinical value for the early detection of NAC efficacy and the decision to shift to intensive treatments for patients with TNBC with poor response to chemotherapy. However, the correlation between the two indicators is weak, which may be because there may be an indirect regulatory relationship between the two indicators.

The tumor microenvironment is composed of tumor cells, immune cells, stromal cells, and other cellular components and their secreted cytokines. It is regulated by the signaling pathways of tumor oncogene activation and/or tumor suppressor gene inactivation, and is related to tumor, Invasion, metastasis and 
treatment tolerance are closely related. Previous work of our research group showed that knocking out UBR5 from the 4T1 TNBC cell line inhibits the expression of E-cadherin, changes the inherent epithelial characteristics of tumor cells, inhibits mesenchymal epithelial transformation, and inhibits distant metastasis of breast cancer. Additionally, by reconstructing the tumor microenvironment, knocking out UBR5 can reduce Treg infiltration, increase DC and CD8+ T cell infiltration, activate anti-tumor immunity, inhibit tumor growth or completely eliminate tumors. These results suggest that UBR5 regulates the tumor microenvironment in TNBC and promotes the invasion and metastasis of TNBC and could become a new therapeutic target for TNBC. However, the mechanism by which UBR5 modulates the TNBC tumor microenvironment is not clear and requires further investigation ${ }^{[37]}$.

Bioinformatics analysis revealed that the expression of UBR5 was highly correlated with the expression of genes such as TMEM184C, SMAD2, TGFBRAP1, BMPR2, NFAT5, REL, SSH1, TNFSF12, and VAMP5. These genes are highly related to immune cell infiltration and immune regulatory pathways. However, there is still no reliable evidence to prove the correlation between these genes and the degree of sTILs infiltration. We also found that the expression of UBR5 was negatively correlated with immune scores. Based on the above evidence, we can know that UBR5 plays an important role in the regulation of the immune system and the infiltration of immune cells. However, whether UBR5 can indirectly affect the infiltration level of sTILs by influencing the expression of immune-related genes and regulating the immune microenvironment is worthy of further investigation. This adds more information to our continued in-depth study of the regulation mechanism of UBR5 and the tumor microenvironment.

This study has certain defects. Firstly, as a retrospective clinical study, some confounding factors may affect the results of experimental. Second, due to the availability of clinical patient samples, this study is a small sample study, Therefore, in order to achieve a wide range of clinical applications, we need a larger sample size to verify the above conclusions.

\section{Conclusion}

The status of UBR5 expression and STILs infiltration can be used to predict the efficacy of NAC in patients with TNBC. This is of great significance for early evaluation of the efficacy of NAC and the adoption of reasonable clinical measures. However, whether UBR5 can affect sTILs infiltration by regulating immune-related genes requires further investigation. UBR5 overexpression is also associated with chemotherapy resistance in patients with TNBC. Therefore, chemotherapy may be more effective to patients with TNBC following UBR5 knockdown or inhibition. Finally, UBR5 and sTILs are also independent factors for DFS in patients with TNBC, confirming their importance for predicting the prognosis of clinical patients.

\section{Abbreviations}

NAC: neoadjuvant chemotherapy; TNBC: triple-negative breast cancer; sTILs: stromal tumor infiltrating lymphocytes; PCR: pathological complete response; NAC: neoadjuvant chemotherapy; DFS: disease-free 
survival; ER: estrogen receptor; PR: progesterone receptor; HER2: human epidermal growth factor receptor 2; DCIS: ductal carcinoma in situ; HR: hazard ratio; Cl: confidence interval; CTC: circulating tumor cell

\section{Declarations}

\section{Ethics approval and consent to participate}

The experimental protocol was established, according to the ethical guidelines of the Helsinki Declaration and was approved by the Human Ethics Committee of Xiangya Hospital. Written informed consent was obtained from individual or guardian participants.

\section{Consent for publication}

Not applicable.

\section{Availability of data and material}

The datasets generated during and/or analyses during the current study are available in the TCGA database.

\section{Competing interests}

The authors declare that there is no conflict of interests regarding the publication of this paper.

\section{Funding}

Not applicable.

\section{Authors' contributions}

Liqiu Liao and Shouman Wang designed and supervised the experimental plan. Jian Pang completed the writing of the article and the analysis and arrangement of experimental data. Li Zhou completed the collection of experimental data. All authors read and approved the final manuscript.

\section{Acknowledgments}

The authors thank AiMi Academic Services (www.aimieditor.com) for English language editing and review services.

\section{Clinical Practice Points}

Neoadjuvant chemotherapy (NAC) has been widely used in patients with TNBC and has important clinical value for predicting the efficacy of early NAC treatment to screen out patients with poor response to chemotherapy. In the tumor tissues of TNBC patients, the expression level of UBR5 and the degree of infiltration of stromal tumor infiltrating lymphocytes (sTILs) can predict the efficacy of chemotherapy, 
and there was a negative correlation between them. Therefore, the combination of these two indicators will improve the ability to predict the efficacy of NAC in patients. Bioinformatics analysis shows that the expression of UBR5 is closely related to immune cells, genes regulated by immune pathways, and immune scores. Thus, exploration of the relationship between UBR5 expression and the tumor microenvironment is worthy of further investigation.

\section{References}

[1] Hu M-H, Wu T-Y, Huang Q, Jin Guangyi. New substituted quinoxalines inhibit triple-negative breast cancer by specifically downregulating the c-MYC transcription[J]. Nucleic acids research.2019; doi:10.1093/nar/gkz835.

[2] Zagorac I, Fernandez-Gaitero S, Penning R, Post H, Bueno M J, Mouron S, et al. In vivo phosphoproteomics reveals kinase activity profiles that predict treatment outcome in triple-negative breast cancer[J]. Nature communications. 2018; doi:10.1038/s41467-018-05742-z.

[3] Sikov W M, Berry D A, Perou C M, Singh B, T Cirrincione C, Tolaney S M, et al. Impact of the addition of carboplatin and/or bevacizumab to neoadjuvant once-per-week paclitaxel followed by dose-dense doxorubicin and cyclophosphamide on pathologic complete response rates in stage II to III triple-negative breast cancer: CALGB 40603 (Alliance)[J]. Journal of clinical oncology: official journal of the American Society of Clinical Oncology. 2015; doi:10.1200/JC0.2014.57.0572.

[4] Dushyanthen S, Teo Z L, Caramia F, Savas P, Mintoff C P, Virassamy B, et al. Agonist immunotherapy restores $T$ cell function following MEK inhibition improving efficacy in breast cancer[J]. Nature communications. 2017; doi:10.1038/s41467-017-00728-9.

[5] Salgado R, Denkert C, Demaria S, Sirtaine N, Klauschen F, Pruneri G, et al. The evaluation of tumorinfiltrating lymphocytes (TILs) in breast cancer: recommendations by an International TILs Working Group 2014[J]. Annals of oncology: official journal of the European Society for Medical Oncology. 2015; doi:10.1093/annonc/mdu450.

[6] Pujani M, Jain H, Chauhan V, Agarwal C, Singh K, Singh M. Evaluation of Tumor infiltrating lymphocytes in breast carcinoma and their correlation with molecular subtypes, tumor grade and stage[J]. Breast disease. 2020; doi:10.3233/BD-200442.

[7] Callaghan M J, Russell A J, Woollatt E, Sutherland G R, Sutherland R L, Watts C K. Identification of a human HECT family protein with homology to the Drosophila tumor suppressor gene hyperplastic discs[J]. Oncogene. 1998; doi:10.1038/sj.onc.1202249.

[8] Henderson M J, Russell A J, Hird S, Muñoz M, Clancy J L, Lehrbach G M. EDD, the human hyperplastic discs protein, has a role in progesterone receptor coactivation and potential involvement in DNA damage response[J]. The Journal of biological chemistry. 2002; doi:10.1074/jbc.M203527200. 
[9] Henderson M J, Munoz M A, Saunders D N, Clancy J L, Russell A J, Williams B, et al. EDD mediates DNA damage-induced activation of CHK2[J]. The Journal of biological chemistry. 2006;

doi:10.1074/jbc.M602818200.

[10] Brotin E, Meryet-Figuiere M, Simonin K, Duval R E, Villedieu M, Leroy-Dudal J, et al. Bcl-XL and MCL-1 constitute pertinent targets in ovarian carcinoma and their concomitant inhibition is sufficient to induce apoptosis[J]. International journal of cancer. 2010; doi:10.1002/ijc.24787.

[11] Shearer R F, Iconomou M, Watts C K W, Saunders D N. Functional Roles of the E3 Ubiquitin Ligase UBR5 in Cancer[J]. Molecular cancer research: MCR. 2015; doi:10.1158/1541-7786.MCR-15-0383.

[12] Qiao X, Liu Y, Prada M L, Mohan A K, Gupta A, Jaiswal A, et al. UBR5 Is Coamplified with MYC in Breast Tumors and Encodes an Ubiquitin Ligase That Limits MYC-Dependent Apoptosis[J]. Cancer research. 2020; doi:10.1158/0008-5472.CAN-19-1647.

[13] Chen L, Yuan R, Wen C, Liu T, Feng Q, Deng X, et al. E3 ubiquitin ligase UBR5 promotes pancreatic cancer growth and aerobic glycolysis by downregulating FBP1 via destabilization of $\mathrm{C} / \mathrm{EBPa}[\mathrm{J}]$. Oncogene. 2020; doi:10.1038/s41388-020-01527-1.

[14] Song $\mathrm{M}$, Wang $\mathrm{C}$, Wang $\mathrm{H}$, Zhang $\mathrm{T}$, Li J, Benezra R, et al. Targeting ubiquitin protein ligase E3 component N-recognin 5 in cancer cells induces a CD8+ T cell mediated immune response[J]. Oncoimmunology. 2020; doi:10.1080/2162402X.2020.1746148.

[15] Loi S, Drubay D, Adams S, Pruneri G, Francis P A, Lacroix-Triki M, et al. Tumor-Infiltrating Lymphocytes and Prognosis: A Pooled Individual Patient Analysis of Early-Stage Triple-Negative Breast Cancers[J]. Journal of clinical oncology: official journal of the American Society of Clinical Oncology. 2019; doi:10.1200/JC0.18.01010.

[16] Tian T, Ruan M, Yang W, Shui R. Evaluation of the prognostic value of tumor-infiltrating lymphocytes in triple-negative breast cancers[J]. Oncotarget. 2016; doi:10.18632/oncotarget.10054.

[17] Carbognin L, Pitotto S, Nortilli R, Brunelli M, Nottegar A, Sperduti I, et al. Predictive and Prognostic Role of Tumor-Infiltrating Lymphocytes for Early Breast Cancer According to Disease Subtypes: Sensitivity Analysis of Randomized Trials in Adjuvant and Neoadjuvant Setting[J]. The oncologist. 2016; doi:10.1634/theoncologist.2015-0307.

[18] Aierken N, Shi H-J, Zhou Y, Shao N, Zhang J, Shi Y, et al. High PD-L1 Expression Is Closely Associated With Tumor-Infiltrating Lymphocytes and Leads to Good Clinical Outcomes in Chinese Triple Negative Breast Cancer Patients[J]. International journal of biological sciences. 2017; doi:10.7150/ijbs.20868.

[19] O'loughlin M, Andreu X, Bianchi S, Chemielik E, Cordoba A, Cserni G, et al. Reproducibility and predictive value of scoring stromal tumour infiltrating lymphocytes in triple-negative breast cancer: a multi-institutional study[J]. Breast cancer research and treatment. 2018; doi:10.1007/s10549-018-4825-8. 
[20] Ruan M, Tian T, Rao J, Xu X, Yu B, Yang W, et al. Predictive value of tumor-infiltrating lymphocytes to pathological complete response in neoadjuvant treated triple-negative breast cancers[J]. Diagnostic pathology. 2018; doi:10.1186/s13000-018-0743-7.

[21] Schmid P, Salgado R, Park Y H, Muñoz-Couselo E, Kim S B, Sohn J, et al. Pembrolizumab plus chemotherapy as neoadjuvant treatment of high-risk, early-stage triple-negative breast cancer: results from the phase 1b open-label, multicohort KEYNOTE-173 study[J]. Annals of oncology: official journal of the European Society for Medical Oncology. 2020; doi:10.1016/j.annonc.2020.01.072.

[22] Eryil Maz M K, Mutlu H, ÜNAL B, Salim D K, Musri FY, Coşkun H S. The importance of stromal and intratumoral tumor lymphocyte infiltration for pathologic complete response in patients with locally advanced breast cancer[J]. Journal of cancer research and therapeutics. 2018; doi:10.4103/09731482.174550.

[23] Chae H, Yoo C, Yoon J-A, Lee H J, Kim K-P, Kim J-E, et al. FcrR3A-158 Polymorphism and Stromal Tumor-Infiltrating Lymphocytes and Survival among Patients with Metastatic HER2-Positive Breast Cancer Receiving Trastuzumab-Based Treatment[J]. Journal of breast cancer. 2018; doi:10.4048/jbc.2018.21.1.45.

[24] Yang X, Rao J, Yang W, Shui R. Evaluation of the Predictive and Prognostic Values of Stromal TumorInfiltrating Lymphocytes in HER2-Positive Breast Cancers treated with neoadjuvant chemotherapy[J]. Targeted oncology. 2018; doi:10.1007/s11523-018-0602-1.

[25] Liu J, Xu Y, Yu M, Liu Z, Xu Y, Ma G, et al. Increased Stromal Infiltrating Lymphocytes are Associated with Circulating Tumor Cells and Metastatic Relapse in Breast Cancer Patients After Neoadjuvant Chemotherapy[J]. Cancer management and research. 2019; doi:10.2147/CMAR.S220327.

[26] Zhang Z, Zheng X, Li J, Duan J, Cui L, Yang L, et al. Overexpression of UBR5 promotes tumor growth in gallbladder cancer via PTEN/PI3K/Akt signal pathway[J]. Journal of cellular biochemistry. 2019; doi:10.1002/jcb.28431.

[27] Wang K, Tang J, Liu X, Wang Y, Chen W, Zheng R. UBR5 regulates proliferation and radiosensitivity in human laryngeal carcinoma via the p38/MAPK signaling pathway[J]. Oncology reports. 2020; doi:10.3892/or.2020.7620.

[28] Saurabh K, Shah P P, Doll M A, Siskind L J, Beverly L J. UBR-box containing protein, UBR5, is overexpressed in human lung adenocarcinoma and is a potential therapeutic target[J]. BMC cancer. 2020; doi: 10.1186/s12885-020-07322-1.

[29] Ding F, Zhu X, Song X, Yuan P, Ren L, Chai C, et al. UBR5 oncogene as an indicator of poor prognosis in gastric cancer[J]. Experimental and therapeutic medicine. 2020; doi:10.3892/etm.2020.9135. 
[30] Yang M, Jiang N, Cao Q-W, Ma M-Q, Sun Q. The E3 ligase UBR5 regulates gastric cancer cell growth by destabilizing the tumor suppressor GKN1[J]. Biochemical and biophysical research communications. 2016; doi:10.1016/j.bbrc.2016.08.170.

[31] Wang J, Zhao X, Jin L, Wu G, Yang Y. UBR5 Contributes to Colorectal Cancer Progression by Destabilizing the Tumor Suppressor ECRG4[J]. Digestive diseases and sciences. 2017; doi:10.1007/s10620-017-4732-6.

[32] Xie Z, Liang H, Wang J, Xu X, Zhu Y, Guo A, et al. Significance of the E3 ubiquitin protein UBR5 as an oncogene and a prognostic biomarker in colorectal cancer[J]. Oncotarget. 2017; doi:10.18632/oncotarget.22531.

[33] Ji S Q, Zhang Y X, Yang B H. UBR5 promotes cell proliferation and inhibits apoptosis in colon cancer by destablizing P21[J]. Die Pharmazie. 2017; doi:10.1691/ph.2017.7433.

[34] Yang Y, Zhao J, Mao Y, Li F, Jiang Z. UBR5 over-expression contributes to poor prognosis and tamoxifen resistance of ERa+ breast cancer by stabilizing $\beta$-catenin[J]. Breast cancer research and treatment. 2020; doi:10.1007/s10549-020-05899-6.

[35] Bian P, Dou Z, Jia Z, Li W, Pan D. Activated Wnt/ $\beta$-Catenin signaling contributes to E3 ubiquitin ligase EDD-conferred docetaxel resistance in prostate cancer[J]. Life sciences. 2020; doi:10.1016/j.Ifs.2019.116816.

[36] Matsuura K, Huang N-J, Cocce K, Zhang L, Kornbluth S. Downregulation of the proapoptotic protein MOAP-1 by the UBR5 ubiquitin ligase and its role in ovarian cancer resistance to cisplatin[J]. Oncogene. 2017; doi:10.1038/onc.2016.336.

[37] Liao L, Song M, Li X, Tang L, Zhang T, Zhang L, et al. E3 Ubiquitin Ligase UBR5 Drives the Growth and Metastasis of Triple-Negative Breast Cancer[J]. Cancer research. 2017; doi:10.1158/0008-5472.CAN-162409.

\section{Tables}

Table 1. Association between UBR5 expression, sTILs infiltration, and clinicopathological factors in patients with TNBC 


\begin{tabular}{|c|c|c|c|c|c|c|}
\hline \multirow[t]{2}{*}{ Characteristics } & \multicolumn{2}{|c|}{ Expression of UBR5 } & \multirow[t]{2}{*}{$p$ value } & \multicolumn{2}{|c|}{ sTILs Infiltration } & \multirow[t]{2}{*}{$\mathrm{p}$-value } \\
\hline & low & high & & low & high & \\
\hline \multicolumn{7}{|l|}{ Tumor size } \\
\hline$\leq 5$ & $39(88.6)$ & $123(87.2)$ & 0.757 & $97(85.1)$ & $65(91.5)$ & 0.195 \\
\hline$>5$ & $5(11.4)$ & $18(12.8)$ & & $17(14.9)$ & $6(8.5)$ & \\
\hline \multicolumn{7}{|l|}{ LN status } \\
\hline pNO & $37(84.1)$ & 104(73.8) & 0.160 & $86(75.4)$ & $55(77.5)$ & 0.753 \\
\hline pN1-3 & $7(15.9)$ & $37(26.2)$ & & $28(24.6)$ & $16(22.5)$ & \\
\hline \multicolumn{7}{|l|}{ Grade } \\
\hline $\mathbb{Q}-\mathbb{\square}$ & $26(59.1)$ & $79(56.0)$ & 0.720 & $70(61.4)$ & $35(49.3)$ & 0.106 \\
\hline ૫ & $18(40.9)$ & $62(44.0)$ & & $44(38.6)$ & $36(50.7)$ & \\
\hline \multicolumn{7}{|l|}{$\mathrm{CD} 8+\mathrm{T}$} \\
\hline Low & $16(36.4)$ & $83(58.9)$ & 0.009 & $63(55.3)$ & $36(50.7)$ & 0.545 \\
\hline High & $28(63.6)$ & $58(41.1)$ & & $51(44.7)$ & $35(49.3)$ & \\
\hline \multicolumn{7}{|l|}{$\mathrm{KI} 67 \%$} \\
\hline$<50$ & $25(56.8)$ & $78(55.3)$ & 0.861 & $69(60.5)$ & $34(47.9)$ & 0.092 \\
\hline$\geq 50$ & $19(43.2)$ & $63(44.7)$ & & $45(39.5)$ & $37(52.1)$ & \\
\hline \multicolumn{7}{|l|}{ Chemosensitivity } \\
\hline PCR & $8(33.3)$ & $5(8.8)$ & 0.016 & $4(7.7)$ & $9(31.0)$ & 0.010 \\
\hline Non-pCR & $16(66.7)$ & $52(91.2)$ & & 48(92.3囚 & $20(69.0)$ & \\
\hline
\end{tabular}

UBR5 and sTILs are factors influencing PCR in patients with TNBC treated with NAC Table 2. Analysis of factors affecting PCR in NAC-treated patients with TNBC 


\begin{tabular}{|c|c|c|c|}
\hline Characteristics & Number of Non-pCR patients & Number of pCR patients & P-value \\
\hline \multicolumn{4}{|l|}{$\mathrm{T}(\mathrm{cm})$} \\
\hline$\leq 5$ & $49(72.1)$ & 11(84.6) & 0.497 \\
\hline$>5$ & 19(27.9) & $2(15.4)$ & \\
\hline \multicolumn{4}{|l|}{ LN } \\
\hline pNO & $44(64.7)$ & $12(92.3)$ & 0.055 \\
\hline pN1-3 & 24(35.3) & $1(7.7)$ & \\
\hline \multicolumn{4}{|l|}{ Grade } \\
\hline 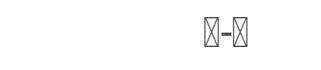 & $40(58.8)$ & $6(46.2)$ & 0.398 \\
\hline प & $28(41.2)$ & $7(53.8)$ & \\
\hline \multicolumn{4}{|l|}{$\mathrm{KI} 167 \%$} \\
\hline$<50$ & $36(52.9)$ & $4(30.8)$ & 0.143 \\
\hline$\geq 50$ & $32(47.1)$ & $9(69.2)$ & \\
\hline \multicolumn{4}{|l|}{ UBR5 } \\
\hline Low & $16(23.5)$ & $8(61.5)$ & 0.016 \\
\hline High & $52(76.5)$ & $5(38.5)$ & \\
\hline \multicolumn{4}{|l|}{ sTILs } \\
\hline Low & $48(70.6)$ & $4(30.8)$ & 0.010 \\
\hline High & $20(29.4)$ & $9(69.2)$ & \\
\hline \multicolumn{4}{|l|}{$\mathrm{CD} 8+\mathrm{T}$} \\
\hline Low & 28(41.2) & $9(69.2)$ & 0.063 \\
\hline High & $40(58.8)$ & $4(30.8)$ & \\
\hline Total & 68 & 13 & \\
\hline
\end{tabular}

UBR5 and sTILs are factors influencing whether patients with TNBC undergoing NAC treatment achieve PCR

Table 3. Multivariate analysis of PCR in NAC-treated patients with TNBC 


\begin{tabular}{|lllllll|}
\hline & B & SE & Wald & P value & $\operatorname{Exp}(B)$ & $95 \%$ Cl of $\operatorname{Exp}(B)$ \\
\hline UBR5 & -1.371 & 0.666 & 4.241 & $\mathbf{0 . 0 3 9}$ & 0.254 & $0.069-0.936$ \\
\hline STILs & 1.427 & 0.683 & 4.366 & $\mathbf{0 . 0 3 7}$ & 4.166 & $1.093-15.887$ \\
\hline
\end{tabular}

UBR5 and STIL are independent factors influencing whether patients with TNBC undergoing NAC treatment can achieve PCR

Table 4. Analysis of factors affecting DFS in patients with TNBC who received NAC treatment but did not reach PCR

\begin{tabular}{|c|c|c|c|c|c|}
\hline \multirow[t]{2}{*}{ Factor } & & \multirow{2}{*}{$\begin{array}{l}\text { Univariate } \\
\text { p-value }\end{array}$} & \multicolumn{3}{|c|}{ Multivariate } \\
\hline & & & $\mathrm{HR}$ & $95 \% \mathrm{Cl}$ & P-value \\
\hline T stage & $(>5 \mathrm{~cm}$ vs. $\leq 5 \mathrm{~cm})$ & 0.003 & 2.640 & $1.165-5.981$ & 0.020 \\
\hline pN stage & (pN1-3 vs. pN0) & 0.001 & 2.949 & $1.298-6.697$ & 0.010 \\
\hline Grade & (\vs. I-II ) & 0.627 & & & \\
\hline $\mathrm{K} 167 \%$ & $(\geq 50$ vs. $<50)$ & 0.702 & & & \\
\hline UBR5 & (high vs. low) & 0.007 & 4.360 & $1.002-18.968$ & 0.050 \\
\hline $\mathrm{CD} 8+\mathrm{T}$ & (high vs. low) & 0.650 & & & \\
\hline sTILs & (high vs. low) & 0.091 & & & \\
\hline
\end{tabular}

UBR5 and sTILs are not independent influencing factors of DFS in patients receiving NAC treatment but not PCR

Table 5. Factors Influencing DFS in patients with TNBC

\begin{tabular}{|llllll|}
\hline Factor & & Univariate & \multicolumn{2}{l|}{ Multivariate } \\
& & p-value & HR & $95 \% \mathrm{Cl}$ & P-value \\
\hline T stage & $(>5 \mathrm{~cm}$ vs. $\leq 5 \mathrm{~cm})$ & $\mathbf{0 . 0 0 2}$ & 2.466 & $1.235-4.923$ & $\mathbf{0 . 0 1 1}$ \\
\hline pN stage & (pN1-3 vs. pN0 $)$ & $\mathbf{0 . 0 0 4}$ & 2.070 & $1.123-3.814$ & $\mathbf{0 . 0 2 0}$ \\
\hline Grade & ( vs. I-II $)$ & 0.574 & & & \\
KI67\% & ( $\geq 50$ vs. $<50)$ & 0.265 & & & \\
UBR5 & (high vs. low) & $\mathbf{0 . 0 0 4}$ & 3.356 & $1.194-9.433$ & 0.022 \\
\hline CD8+T & (high vs. low) & 0.952 & & & \\
\hline sTILs & (high vs. low) & $\mathbf{0 . 0 0 3}$ & 0.401 & $0.193-0.836$ & $\mathbf{0 . 0 1 5}$ \\
\hline
\end{tabular}


ubr5, sTILs, lymph node staging and tumor size are independent influencing factors of DFS

Table 6. Correlation between UBR5 expression and sTILs infiltration in patients with TNBC

\begin{tabular}{|llllll|}
\hline Characteristics & \multicolumn{3}{c}{ sTILs } & r & P-value \\
\hline \multirow{3}{*}{ UBR5 } & Low & High & & \\
& Low & 20 & 24 & -0.186 & $\mathbf{0 . 0 1 1}$ \\
\cline { 2 - 6 } & High & 94 & 47 & & \\
\hline
\end{tabular}

The expression of UBR5 is negatively correlated with the degree of infiltration of sTILs in patients with $\operatorname{TNBC}(r=-0.186 ; p=0.011)$

\section{Figures}

\section{Primary breast cancer( $\mathrm{n}=199)$}

- Breast cancer during pregnancy $(n=3)$

- Distant metastasis at first diagnosis $(n=6)$

- Patients with acute and chronic inflammatory $(n=5)$
PCR patients $(\mathrm{n}=13)$

Non-PCR patients $(\mathrm{n}=68)$

\section{Invasive breast cancer $(\mathrm{n}=185)$}

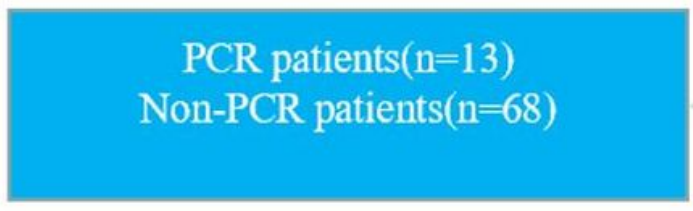

Neoadjuvant chemotherapy patients $(\mathrm{n}=81)$

\section{Figure 1}

Patient inclusion flowchart. 

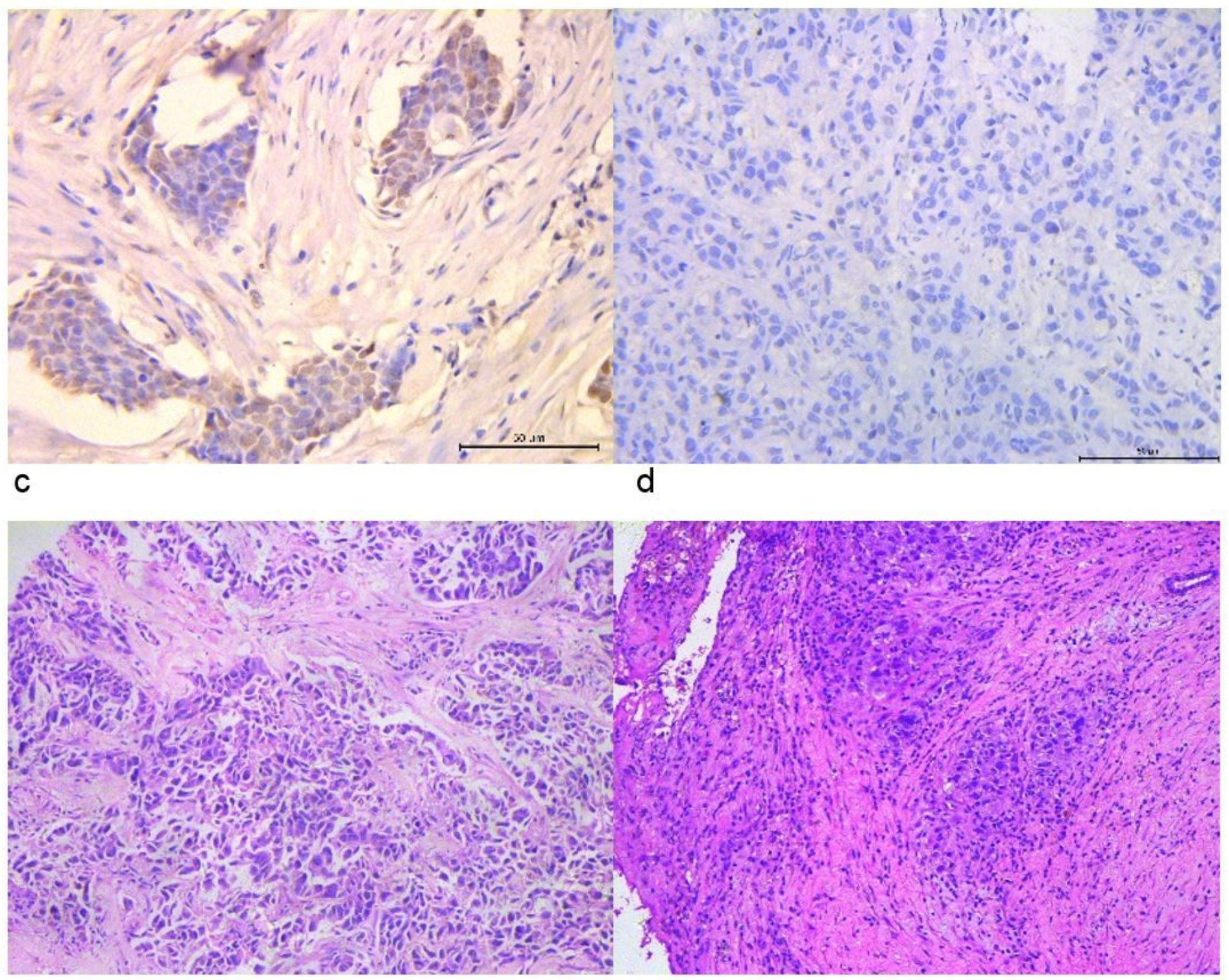

\section{Figure 2}

Immune tissue staining and evaluation of sTILs infiltration. (a) UBR5 positive expression (b) UBR5 negative expression (c) Low sTILs score (0-10\% sTILs). (d) High sTILs score (> 10\% sTILs). 
a

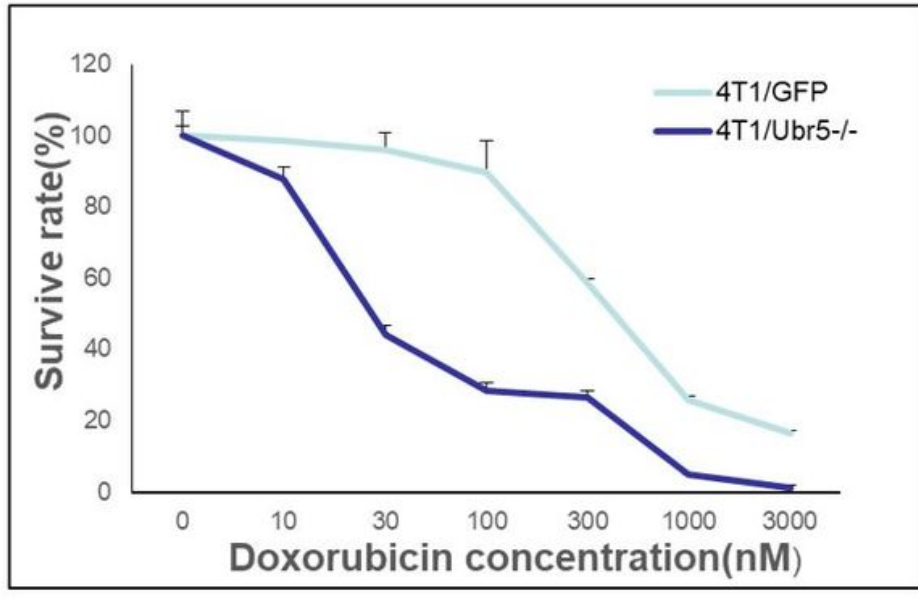

b

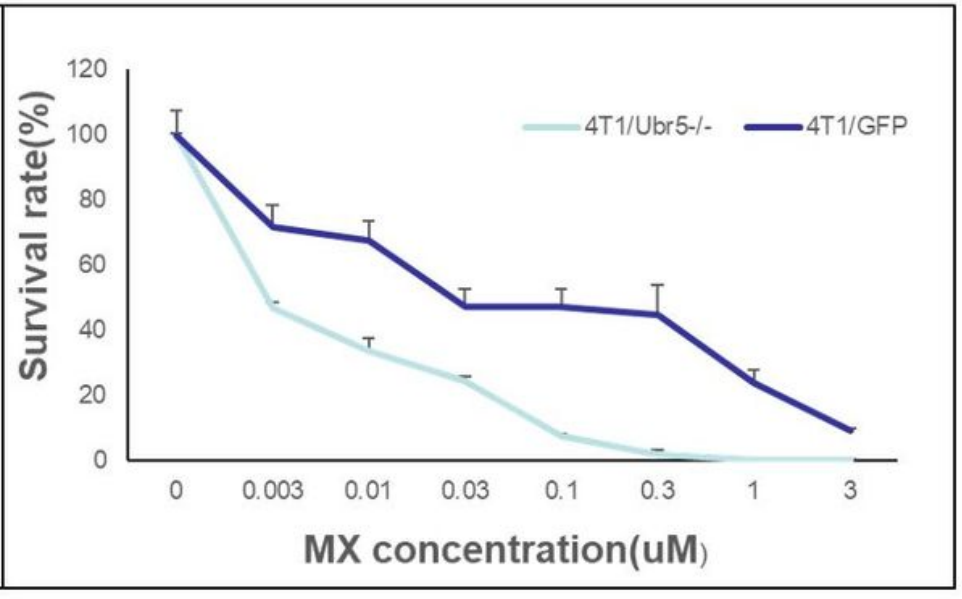

Figure 3

Drug sensitivity test. (a) Determination of the sensitivity of TNBC cells to the chemotherapy drug doxorubicin. (b) Determination of the sensitivity of TNBC cells to the chemotherapy drug mitoxantrone $(\mathrm{MX})$. 
b
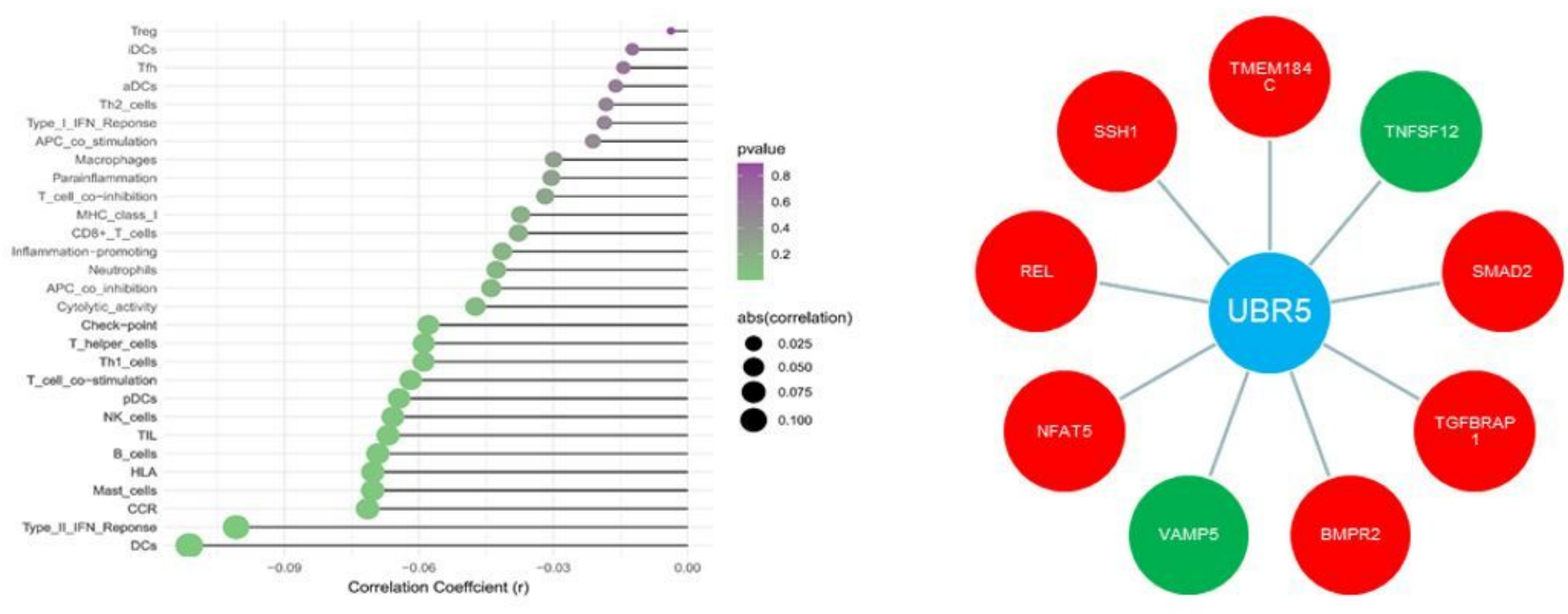

C
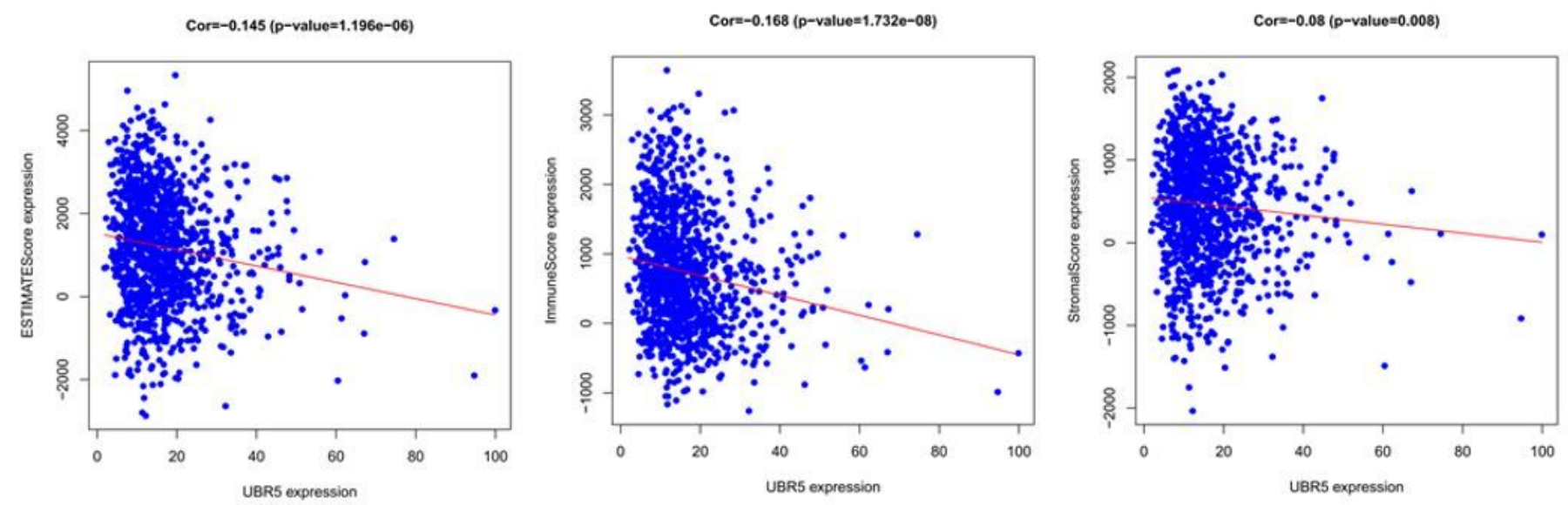

Figure 4

(4a) The correlation between UBR5 and infiltrating immune cells. The size of the dot represents the strength of the correlation between genes and immune cells; the larger the dot, the stronger the correlation, and the smaller the dot, the weaker the correlation. The color of the dot indicates the $p$ value. The greener the color, the lower the p-value, the more purple the color, the larger the p-value. The bold font on the left represents the infiltrating immune cells or immunoregulatory pathways related to the expression of UBR5 in triple-negative breast cancer. $p<0.05$ was considered statistically significant. (4b) The relationship between UBR5 and immune-related genes. The red circle represents a positive correlation between UBR5 expression and its gene expression. The green circle represents that UBR5 expression is negatively correlated with this gene expression. (4c) The correlation between UBR5 and immune score.

\section{Supplementary Files}

This is a list of supplementary files associated with this preprint. Click to download. 
- SupplementaryData.pdf

Page 22/22 